\title{
Molecular Characterization and Expression of Synaptic Plasticity-related Genes in the Olfactory Organ and Brain of Chum and Pink Salmon during Seaward and Homeward Migration
}

\author{
Takashi Abe* and Hideaki Kudo \\ Laboratory of Humans and the Ocean, Faculty of Fisheries Sciences, Hokkaido University, Hakodate 041-8611, \\ Japan \\ *Present affiliation: Shibetsu Salmon Museum, Shibetsu 086-1141, Japan
}

Keywords: Anadromous, Homing, Imprinting, Olfaction, Neuroplasticity

Homeward migration of anadromous Pacific salmon (Genus Oncorhynchus) depends on the imprinted memory of odorants from their natal streams prior to seaward migration. Previous studies suggested the importance of synaptic plasticity in the olfactory imprinting of salmon. Although some neurotransmitters and receptors suggested its regulatory function in the olfactory imprinting by salmon, the relationship between pre-synapse molecules and olfactory imprinting was not revealed. A soluble N-ethylmaleimide-sensitive factor attachment protein receptor (Snare) complex mediates vesicle exocytosis in pre-synapse and regulates synaptic transmission and neuroplasticity (Fig. 1). In this study, three Snare component genes; snap25, stxl, and vamp2 were isolated from the olfactory center (olfactory bulb [OB] and telencephalon) of both chum salmon (Oncorhynchus keta) and pink salmon (Oncorhynchus gorbuscha) and the expressions in the olfactory nervous system during migrations of these salmon were analyzed by molecular biological techniques.
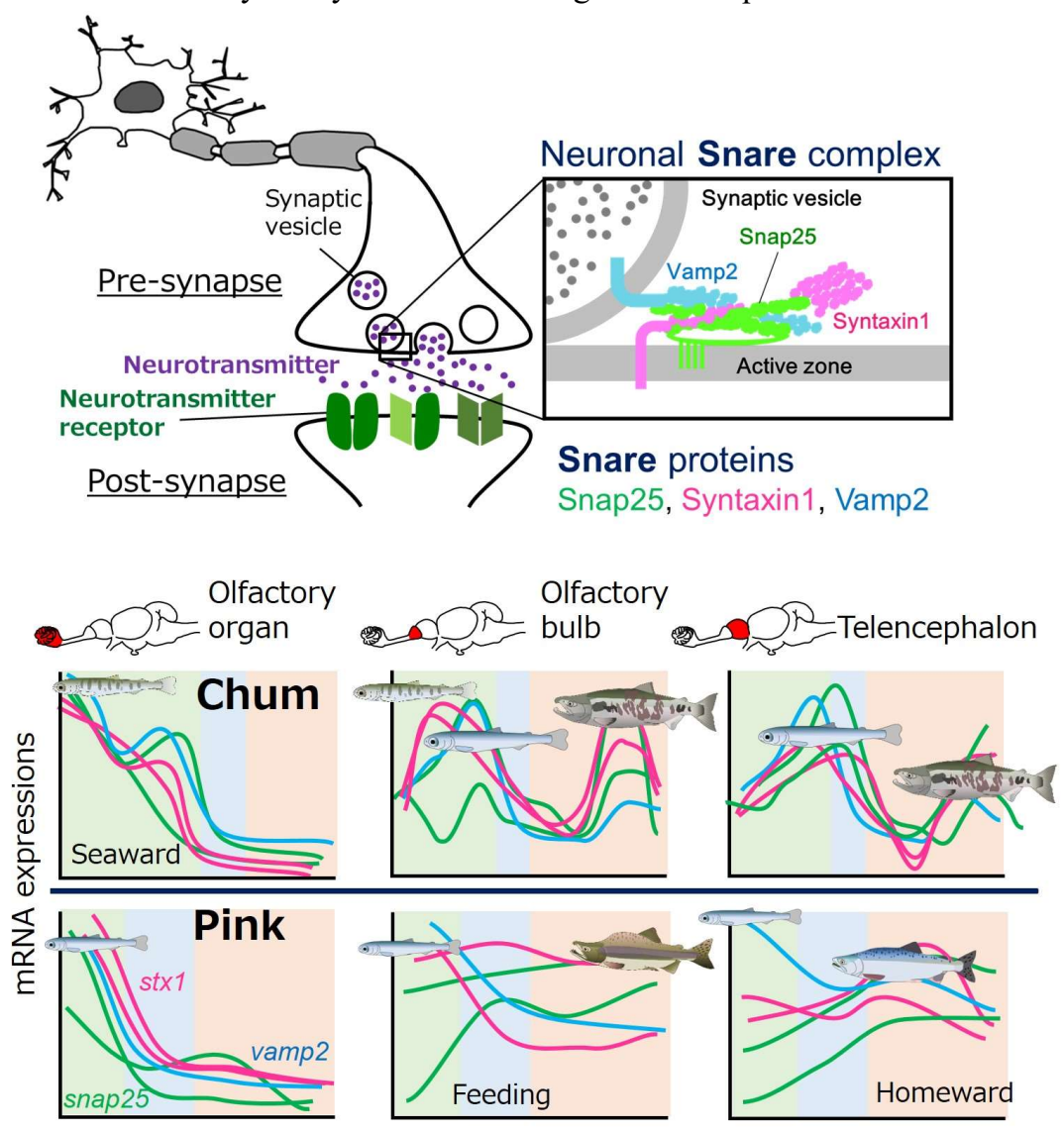

Fig. 1. Upper image: Schematic illustration of synaptic-vesicle exocytosis and its regulating protein complex soluble N-ethylmaleimidesensitive factor attachment protein receptor (Snare). Lower image: Snare mRNA expressions in olfactory nervous system of chum and pink salmon. Snare: soluble Nethylmaleimide-sensitive factor attachment protein receptor; Snap25: synaptosome-associated protein 25 ; Stx1: syntaxin 1; Vamp2: vesicleassociated membrane protein 2 .

Molecular characterization of cDNA encoding salmon Snap25 indicated that alternative splicing produced both salmon Snap25a and Snap25b, as known in high vertebrate species. Two Syntaxin isoforms, stx $1 a$ and stx $1 b$, were isolated as single-copy genes according to expectation. Salmon Vamp2 (110 residues) was shorter than that of high vertebrate species (115 residues) while the SNARE motif was highly conserved. There was a highly conserved 
amino- and carboxyl-terminal SNARE motif in Snap25s, H3 domain including SNARE motif in Stx1s, and RSNARE motif in Vamp2 between chum, pink, and the other vertebrates. Their phylogenies showed similarities of molecular evolution of Snare deduced amino acids between salmonids and other vertebrates.

Through quantitative-PCR analyses of the chum salmon olfactory organ (olfactory rosette: OR) and brain, these SNAREs mRNA (snares) showed similar expression patterns in each brain region as seen in other vertebrate species. In chum salmon, snare mRNA in the OR expressed significantly more during seaward migration than in adult life stages, including feeding and homeward migration. High snare mRNA expressions in a juvenile's OR reflected the development of the olfactory nervous system. Snare mRNA in the OB (primarily olfactory center) showed the highest expression levels during seaward migration of juveniles. Snap $25 b$ and stx $1 s$ in the telencephalon (including higher olfactory center) increased not only during the seaward period but also in the homeward adult. Vamp2 in the telencephalon decreased in adults as in the OB.

In pink salmon, snare mRNA expression levels remained higher in the OR during homeward migration and decreased during upriver migration. These profiles were related closer to two adjacent periods, the development of the olfactory nervous system and homeward migration, than in chum salmon. In pink salmon, the developmental period of the olfactory nervous system and sexual maturation are adjacent to each other, therefore snare mRNA levels remained higher during the early phase of homeward migration. In the $\mathrm{OB}$, snap $25 b$ increased while $s t x 1 b$ and vamp 2 decreased. In the telencephalon, snap $25 s$ and stx $1 a$ increased in adults while vamp 2 decreased as in chum salmon.

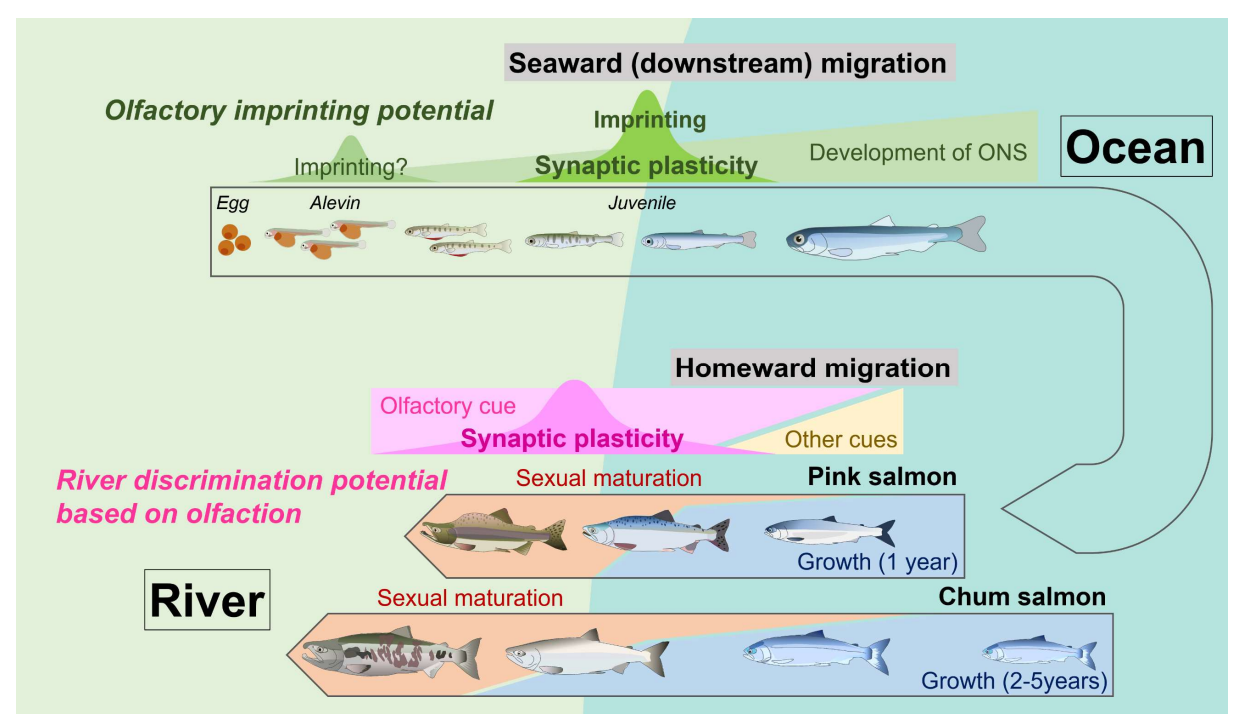

Fig. 2. Schematic illustration indicates synaptic plasticity in the olfactory nervous system and related physiological changes and environmental cues in both seaward and homeward migration of chum salmon (Oncorhynchus keta) and pink salmon (Oncorhynchus gorbuscha). OSN: olfactory nervous system.

In these two Pacific salmon species snares mRNA was regulated similarly (Fig. 1). That pattern was upregulation during seaward migration and temporal upregulation during homeward migration. However, snares regulation in adults showed differences between the two salmon species. This is possibly related to sexual maturation age in each species: mainly three-five years in chum, compared with two years in pink salmon. In addition, migration patterns and dependence on the river for reproduction are known to differ between chum and pink salmon (Fig. 2).

This study evaluated neuronal development and plasticity status of the olfactory nervous system in two Pacific salmon species that have different migratory and maturation features. Imprinting probability is deeply related to reproduction success and enlargement of distribution. In summary, it was suggested that salmon snare mRNA expressions reflected neuronal development in the peripheral OR, possibly associated with primary projection into the $\mathrm{OB}$ and related with higher neural regulation in the telencephalon. In future studies, snare genes will be useful molecular markers to evaluate neuronal development and activity in salmon, coping with changing environment in the Pacific area. 